\title{
Parylene C coated microelectrodes for scanning electrochemical microscopy
}

\author{
Fernando Cortés-Salazara, ${ }^{\mathrm{a}, 1}$, Haiqiang Deng ${ }^{\mathrm{a}}$, Pekka Peljo ${ }^{\mathrm{b}, 1}$, Carlos M. Pereira ${ }^{\mathrm{c}, 1}$, \\ Kyösti Kontturi ${ }^{b}$, Hubert H. Girault ${ }^{\mathrm{a}, *, 1}$ \\ a Laboratoire d'Electrochimie Physique et Analytique, Ecole Polytechnique Federale de Lausanne, CH-1015 Lausanne, Switzerland \\ ${ }^{\mathrm{b}}$ Department of Chemistry, Aalto University, FI-00076 Helsinki, Finland \\ c Centro de Investigação em Química - UP, L4, Departamento de Química e Bioquímica da Faculdade de Ciências, Universidade do Porto, $4169-007$ Porto, \\ Portugal
}

\section{A R T I C L E I N F O}

\section{Article history:}

Available online 6 April 2013

\section{Keywords:}

Parylene $C$ coating

Microelectrode fabrication

Scanning electrochemical microscopy

\begin{abstract}
A B S T R A C T
Herein, we present a simple microelectrode preparation methodology consisting in coating a platinum wire or a carbon fiber with a thin insulating Parylene $C$ film $(e . g .1-10 \mu \mathrm{m})$, to produce SECM probes with a small and constant probe RG (i.e. ratio between the radius of the insulating sheath and the radius of the active electrode area). After exposition of a fresh active electrode area by blade cutting, a disc shaped electrode is obtained thanks to a protective hot mounting wax layer that avoids Parylene $C$ coating deformation and is easily removed with acetone. Stiffness and straightness of the probe can be tuned by modifying the Parylene $C$ coating thickness and the length of the carbon fiber or platinum wire. This simple electrode preparation method is highly reproducible (c.a. $>90 \%$ ). The prepared Parylene $C$ coated microelectrodes were characterized by optical microscopy, cyclic voltammetry, scanning electrochemical microscopy (SECM) approach curves and finally applied to SECM imaging of Pt band structures in contactless and contact mode.
\end{abstract}

(c) 2013 Elsevier Ltd. All rights reserved.

\section{Introduction}

Scanning electrochemical microscopy (SECM) has recently attracted the attention of a broad audience since it has been successfully applied to a wide range of research fields, such as biosensing [1-3], forensic science [4,5], corrosion [6,7], surface patterning $[8,9]$ and catalysis $[10,11]$. The gist of SECM is the use of a scanning microelectrode as a probe to sense locally the reactivity of processes taking place at interfaces such as liquid/liquid, solid/liquid and gas/liquid [12-14]. To perform SECM experiments, a fine positioning system combined with a (bi)potentiostat is required to scan the probe in a very close proximity to the substrate (e.g. at a constant and variable vertical and horizontal direction, respectively), while recording the current at the scanning microelectrode typically biased at a potential where the mass transport controlled electrolysis of a redox species takes place. Thus, the communication between the microelectrode and the interface under study is mediated by redox species that undergo charge transfer processes at the probe and depending on the spatial sample reactivity at the substrate. Therefore, when scanning over

\footnotetext{
* Corresponding author. Tel.: +41 21693 3145; fax: +41 216933667 .

E-mail address: hubert.girault@epfl.ch (H.H. Girault).

1 ISE member.
}

reactive areas able to regenerate the electrolyzed redox species, an increase at the microelectrode current is observed (i.e. positive feedback). On the contrary, low current values are recorded when scanning over non-reactives areas, due to the blocking of the diffusion of the redox mediator to the surface of the microelectrode (i.e. negative feedback). The aforementioned SECM feedback mode is perhaps the most widely used [15], but several other operation modes have been also proposed for localized surface characterization [12,16-18]. Finally, meaningful chemical and electrochemical reactivity information can be derived quantitatively from the SECM response, as it can be described by a mathematical model that comprises the coupling between mass transport and (electro)chemical kinetic aspects $[15,19,20]$.

One of the key points in SECM studies is the micro or submicrometer-sized probe employed to scrutinize the interfacial processes, since it has to fulfill both technical and practical requirements. For instance, different probes have been developed in order to overcome specific SECM limitations, demanding breakthroughs on the state of the art of microelectrode fabrication or a higher level of probe functionalization [21-42]. Typically SECM probes are constructed by encapsulating metallic wires (e.g. Pt, Au) or carbon fibers in a glass capillary, making electrical connections with a conductive paste to a copper wire and exposing a cross-section of the microelectrode by mechanical polishing with different size-particle polishing pads [43]. 
Although glass-encapsulated microelectrodes are the most widely used, their preparation is time consuming, requires some experimental skills and frequently produces electrodes with geometrical defects (e.g. not circular or with active electrode areas shifted from the probe center) that can drastically affect the SECM probe response $[43,44]$. Additionally, preparation of probes with small RG (i.e. ratio between the radius of the insulating sheath and the radius of the active electrode area) values is cumbersome, since extremely precise mechanical grinding or glass etching with $\mathrm{HF}$ is required and not easily repeated when a fresh microelectrode surface is sought $[45,46]$. For these reasons, alternative insulating materials that overcome these drawbacks have been proposed mainly based on polymeric materials that can be deposited by electrophoretic methods, polymerization (i.e. electrochemical, chemical or ultraviolet) or using low melting point materials (e.g. wax) [47-53]. As a result, nano and micrometer probes with welldefined insulating film thicknesses and thus RG values have been reproducible obtained. Moreover, exposing a probe cross-section by cutting with a sharp razor blade or by generating an arc of current at the probe tip generates a microelectrode disk that is localized at the center of the insulating film. For instance, non-invasive carbon fiber electrodes have been successfully localized inside a fine mesh of a commercial glucose sensor to unravel the real affected section of the tested samples during normal glucose analysis [53].

A similar strategy is routinely employed by neuroscientists to prepare single-use implantable microelectrodes and microelectrode arrays with etched metallic wires that are only few micrometers protruding, deliberately exposed or prepared by photolithography protocols [54-59]. Parylene $C$ is a poly(p-xylene) polymer with excellent chemical and mechanical stability, pinhole free coatings and with an established deposition process. Despite the employment of Parylene C coating in SECM related experiments, for instance, on the preparation of $100 \mathrm{~nm}$ thick gold covered-AFM cantilevers for SECM-AFM [29], to insulate semi-ring disc electrodes integrated on glass micropipettes for SECM-SICM experiments [60] or for the preparation of soft stylus probes [21], to the best of our knowledge no attempts have been performed in order to prepare micrometer probes for SECM.

Herein, we present a simple microelectrode microfabrication methodology based on the insulation of metallic wires or carbon fibers previously connected to a copper wire with a well-defined Parylene $C$ film, which allows for round, centered and well defined disc microelectrodes with small RG values. Due to the fact that Parylene $C$ coatings are chemically stable and pinhole free, SECM experiments can be achieved in both aqueous and organic solvents. Moreover, non-straight probes can be prepared easily by shaping the copper electrical contact, for instance to produce submarine electrodes. Fresh and reproducible microelectrode surfaces can be regenerated by blade cutting of a wax-covered Parylene $C$ probe followed by a solvent treatment to remove any wax residues. Cyclic voltammograms, SECM approach curves and SECM imaging were successfully achieved by using these Parylene $C$ microelectrodes.

\section{Experimental}

\subsection{Chemicals}

Ferrocene methanol $\left(\mathrm{FcCH}_{2} \mathrm{OH}, \geq 97 \%\right.$, Sigma-Aldrich $)$ and $\mathrm{KNO}_{3}$ ( $\geq 99 \%$, Buchs, Switzerland) were used as received. Decamethyl ferrocene (DMFc) was purchased from Aldrich. Anhydrous lithium chloride ( $\mathrm{LiCl}$ ), bis(triphenylphosphoranylidene) ammonium chloride $(\mathrm{BACl})$, lithium sulfate $\left(\mathrm{Li}_{2} \mathrm{SO}_{4}\right), 1,2$-dichloroethane (DCE) were obtained from Fluka. Lithium tetrakis(pentafluorophenyl)borate diethyl etherate (LiTB) was purchased from Boulder Scientific and sulfuric acid $\left(\mathrm{H}_{2} \mathrm{SO}_{4}, 95-97 \%\right)$ was purchased from Sigma-Aldrich. $\mathrm{DMFc}^{+} \mathrm{TB}^{-}$in DCE was prepared as reported by Su et al. [61] Briefly a two-phase shake flask reaction between an aqueous phase containing $5 \mathrm{mM}$ LiTB in $50 \mathrm{mM} \mathrm{H}_{2} \mathrm{SO}_{4}$ and a DCE phase with $5 \mathrm{mM}$ DMFc in $5 \mathrm{mM}$ BATB was carried out until the total amount of DMFc was oxidized to $\mathrm{DMFc}^{+}$(c.a. $1 \mathrm{~h}$, verified by steady-state cyclic voltammetry and UV-vis spectroscopy, results not shown). $\mathrm{DMFc}^{+} \mathrm{TB}^{-}$can then be obtained from the isolation of the DCE phase. Bis(triphenylphosphoranylidene) ammonium tetrakis(pentafluoro-phenyl)borate (BATB) was prepared by metathesis of $1: 1$ mixtures of $\mathrm{BACl}$ and $\mathrm{LiTB}$, in a methanol/water $(v / v=2)$ mixture, followed by recrystallization in acetone. Deionized water was produced by a Milli-Q plus 185 model from Millipore (Zug, Switzerland).

\subsection{Preparation of Parylene C coated microelectrodes}

The following protocol was performed in order to prepare the Parylene C coated microelectrodes: (i) electrical connection: a Pt wire (radius $=r_{\mathrm{T}}=12.5 \mu \mathrm{m}$, Goodfellow, Oxford, UK) or a carbon fiber $\left(r_{\mathrm{T}}=5 \mu \mathrm{m}\right.$, Goodfellow, Oxford, UK) is physically connected to a tin coated copper wire by using soldering tin. Special attention has to be paid in order to obtain a straight Pt wire (or carbon fiber). The shape of the probe can be defined by bending the tin coated copper wire, for instance to create a submarine electrode. (ii) Parylene $C$ coating: The complete probe is coated with a Parylene $C$ coating of defined thickness (e.g. 1-10 $\mu \mathrm{m}$ ) by using a Parylene deposition system (Comelec SA, La Chaux-de-Fonds, Switzerland). Briefly, the starting di-p-xylene dimer is sublimated by heat treatment $\left(>100^{\circ} \mathrm{C}\right.$, chamber No. 1$)$ and then cleaved into two p-xylene monomers in a high temperature $\left(>500^{\circ} \mathrm{C}\right)$ pyrolysis chamber (chamber No. 2). Finally room temperature polymerization of the p-xylene monomer over the metallic wire or carbon fiber takes place on a third final chamber. Stiffness and straightness of the probe can be tuned by modifying the Parylene $C$ coating thickness and the length of the Pt wire. (iii) Electrode exposition: To expose a cross section of the Parylene $C$ coated microelectrode, the probe is covered with a hot mounting wax (Allied High Tech Products, East Pacifica Place, United States) that protects mechanically the Parylene $C$ coating during blade cutting and can be easily removed with acetone after washing about 1 min. Similarly, nail varnish can also be used as mechanical protecting layer during blade cutting and then be removed with acetone. The electrodes are slightly protruded after blade cutting, and therefore higher current values than the one obtained with perfectly inlaid microelectrodes might be recorded. $90 \%$ of the prepared probes presented stable and good electrochemical behavior (vide infra), however a $10 \%$ of the probes had an irregular electrochemical response (e.g. high capacitive current and resistance) due to non-uniform Parylene coating and inappropriate manipulation of the probes (e.g. by breaking the electrical connection between the Pt wire and the copper wire). The complete fabrication process can be performed in half of day and several microelectrodes can be prepared at the same time. At the moment 10 probes have been prepare in one run, and the process can be easily scaled up.

\subsection{Preparation of platinum on glass samples}

Pt on glass samples were prepared by following standard photolithographic processes to achieve, for instance, Pt bands with a width, pitch and thickness equal to $50 \mu \mathrm{m}, 50 \mu \mathrm{m}$ and $100 \mathrm{~nm}$, respectively. Positive and negative feedback were tested over a substrate with insulating (glass) and conductive (Pt) regions fabricated in the same manner as the Pt band structure. 


\subsection{SECM experiments}

SECM measurements were carried out using a custom-built SECM setup running under SECMx software [62] and comprising an IVIUM compactstat (IVIUM Technologies, The Netherlands) operating in a classical three-electrode mode. Data analyses were carried out using MIRA software [63]. The electrochemical cell comprises of an $\mathrm{Ag}$ wire as quasi-reference electrode (QRE), a Pt wire as counter electrode (CE) and a Parylene $C$ coated microelectrode as working electrode (WE). All potentials are reported with respect to the Ag QRE. After blade cutting, the quality of the WE was checked with a scanning laser microscope (VK 8700 , Keyence). All the samples were mounted on the bottom of a flat cell construction and investigated at room temperature $\left(20 \pm 2{ }^{\circ} \mathrm{C}\right)$ in a Faraday cage.

The probe was mounted on a common SECM holder at $90^{\circ}$ with respect to the sample surface and approached directly to the substrate for SECM imaging in contact and contact-less mode. When the probe was pressed against the sample during SECM imaging in contact mode, a slight bending of the polymeric probe was observed. No efforts were made in order to define the degree or direction of such bending. However, as reported previously a lift-off routine included in SECMx software was employed for SECM imaging in contact mode [64]. Briefly, during the forward scan the probe is hold at the same vertical positioning value in which a physical contact with the sample substrate is established. In contrast, during the backward scan the probe is lift-off from the sample substrate by a stroke height value where the probe is in a completely contactless situation. After completion of the backward scan, the probe is moved by a pre-defined incremental step in the scanning direction and then pressed against the sample substrate by decreasing the vertical probe position by the same stroke value employed previously. Subsequent scans follow the same procedure. Therefore, SECM images in contact mode were constructed only from the data acquisition of forward line scans.

\section{Results and discussion}

The development of alternative strategies for microelectrode fabrication for SECM is of high relevance in order to overcome some limitations (i.e. time consuming preparation, easy to break upon contact with the sampled substrate and control of geometrical parameters is very demanding and time consuming) of the conventional glass microelectrodes and to fit the requirements of special tasks $[21,37,38,53,65-67]$. The preparation of Parylene C coated microelectrodes fulfill all these points since it is less time consuming (i.e. 10 or more microelectrodes can be prepared in half of a day), presents a high success in preparation and allows to have a well-defined geometry with a constant and small RG value, as shown in Fig. 1. Furthermore, the presented methodology can be extrapolated to the preparation of microelectrodes with different electrode materials (e.g. $\mathrm{Ag}, \mathrm{Au}$ ) by using the same conditions, in contrast to glass-encapsulation based protocols that require the optimization for each electrode material. The latter is thanks to the fact that Parylene C films are highly uniform (see Fig. 1c) and that mechanical deformation of the probe can be avoided during blade cutting by a wax (or nail varnish) protecting layer easily removable by acetone (see Fig. 1d-f). Thus a small and well-defined RG value can be obtained from one cut to the next, which is of high relevance for SECM experiments. Indeed, the probe RG not only affects the response of the microelectrode, but also influences the actual working distance $(d)$ between the probe and the substrate, as big RG probes have a higher probability to crash the substrate if a misalignment between the substrate and probe planes exists. Therefore, it allows bringing the active electrode area of the probe in a very close proximity to the surface. Moreover, due to the polymeric materials employed for the probe preparation, experiments in contact mode are achievable without probe-substrate destruction (vide infra).

To verify the electrochemical response of the Parylene $C$ coated microelectrodes exposed by blade cutting, cyclic voltammetries of $\mathrm{Pt}$ and carbon fiber microelectrodes were performed in aqueous (FcMeOH $2.2 \mathrm{mM}$ and $\mathrm{KNO}_{3} 0.1 \mathrm{M}$ ) and organic media $\left(\mathrm{DMFc}^{+}\right.$ $0.5 \mathrm{mM}$ and BATB $5 \mathrm{mM}$ in DCE). Low capacitive currents and steady-state behavior typical from hemispherical diffusion were observed in all cases, even though the obtained microelectrodes do not present a highly smooth surface or the experiments were carried out in an organic solvent such as DCE (see Fig. 2). Indeed, log plot analysis of the cyclic voltammetries performed at $10 \mathrm{mV} \mathrm{s}^{-1}$ for the Pt and carbon fiber microelectrodes in presence of $\mathrm{FcMeOH}$ and
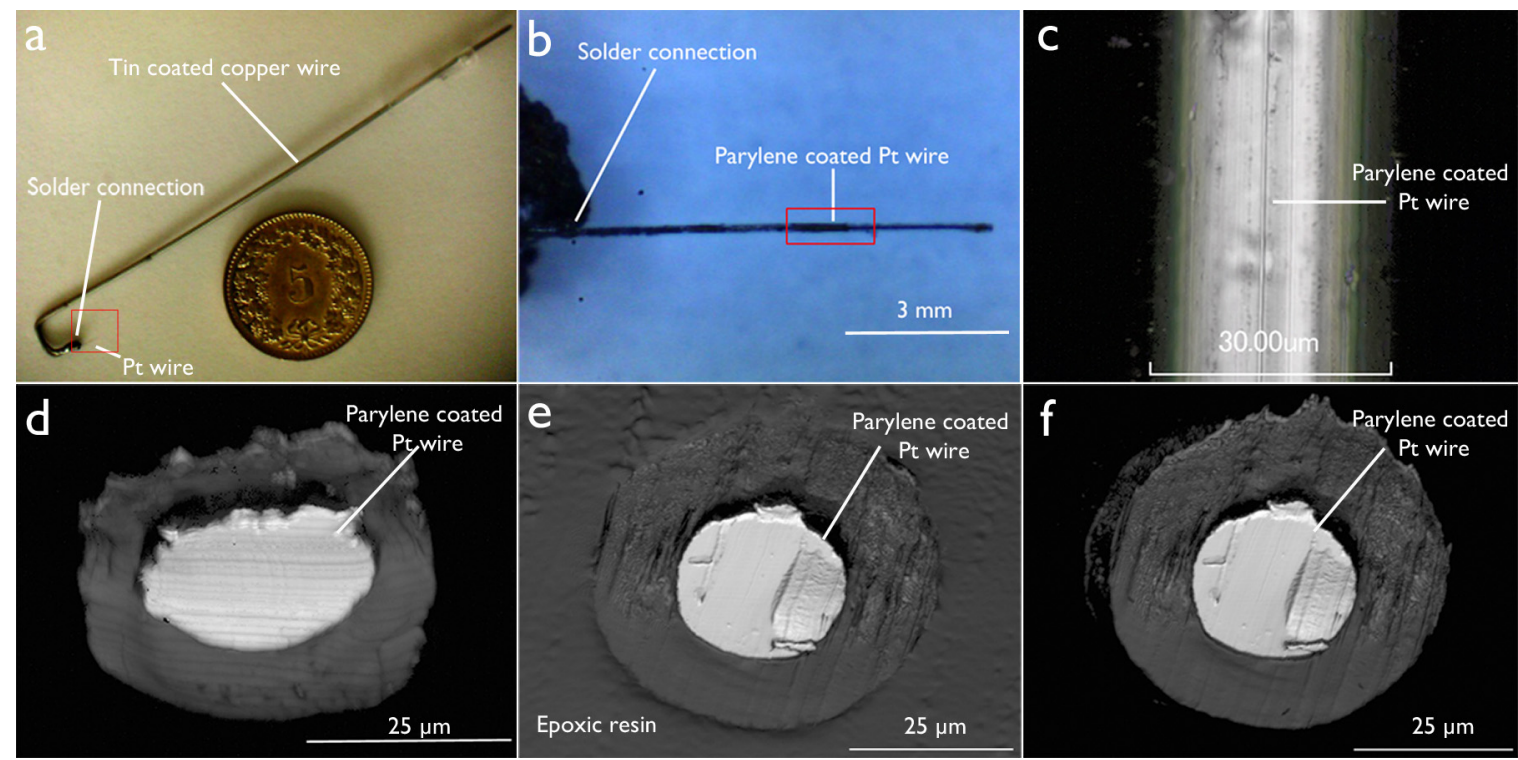

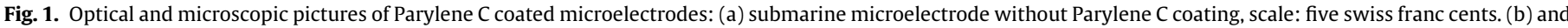

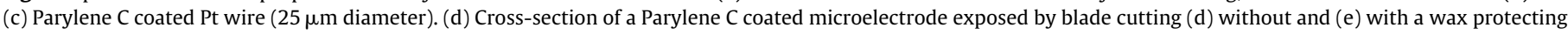
layer. (f) Parylene $\mathrm{C}$ coated microelectrode obtained after washing out the wax protecting layer with acetone. 

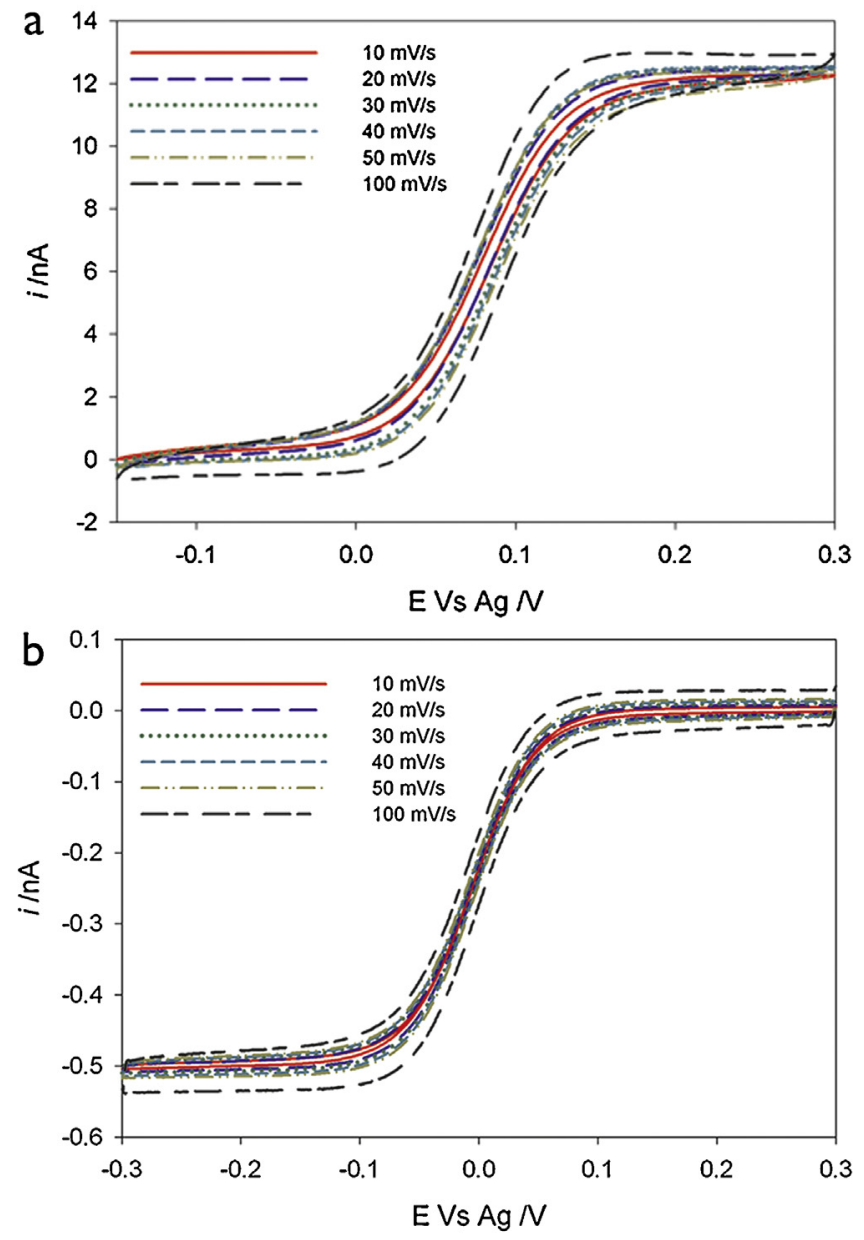

Fig. 2. Cyclic voltammetries of Parylene $C$ coated (a) Pt wire (radius $=12.5 \mu \mathrm{m}$ ) and (b) carbon fiber (radius $=5 \mu \mathrm{m}$ ) at different scan rates. Working conditions: (a) $2 \mathrm{mM}$ of ferrocene methanol in $\mathrm{KNO}_{3} 0.1 \mathrm{M}$, (b) $0.5 \mathrm{mM}$ decamethyl ferrocenium in $5 \mathrm{mM}$ BATB. Scan rates employed: $10 \mathrm{mV} \mathrm{s}^{-1}$ (continuous line), $20 \mathrm{mV} \mathrm{s}^{-1}$ (dashed line), $30 \mathrm{mV} \mathrm{s}^{-1}$ (dotted line), $40 \mathrm{mV} \mathrm{s}^{-1}$ (short dashed line), $50 \mathrm{mV} \mathrm{s}^{-1}$ (dot-dashed line) and $100 \mathrm{mV} \mathrm{s}^{-1}$ (dash-dashed line). Counter electrode $=$ Pt wire, quasi-reference electrode $=$ Ag wire.

$\mathrm{DMFc}^{+}$gave slope values equal to $62.20 \mathrm{mV}$ and $62.55 \mathrm{mV}$, respectively. These values are in very good agreement with the expected one for fast charge transfer processes (i.e. $59.1 \mathrm{mV}$ ).

An interesting feature of small RG value microelectrodes is that a higher steady-state current is normally recorded in comparison with a microelectrode with identical active electrode area, but with a bigger RG value. This is due to the diffusion of redox species from backside the microelectrode plane when the insulating sheath is not big enough to block such process (i.e. back diffusion). To properly calculate the steady-state current $\left(i_{\mathrm{T}, \infty}\right)$ achieved at small RG disc microelectrodes, a correction factor $(\beta)$ that takes into account the back diffusion, as a function of the RG has to be considered, as follows:

$i_{\mathrm{T}}=4 n F D c * r_{\mathrm{T}} \beta$

where $n$ is the number of transferred electrons, $F$ is the Faraday constant, $D$ is the diffusion coefficient of the redox species, $c^{*}$ is the concentration of the redox species and $r_{\mathrm{T}}$ is the radius of the disc microelectrode. By taking into account the respective $\mathrm{RG}$ values for the $\mathrm{Pt}\left(\mathrm{RG}=1.2, r_{\mathrm{T}}=12.5 \mu \mathrm{m}, \beta=1.237\right)$ and carbon fiber ( $\left.R G=1.5, r_{\mathrm{T}}=5 \mu \mathrm{m}, \beta=1.164\right)$ microelectrodes [68], as well as, the diffusion coefficients and concentration of the employed redox species $\left(\mathrm{FcMeOH} D=7.8 \times 10^{-10} \mathrm{~m}^{2} \mathrm{~s}^{-1}, c^{*}=2.2 \mathrm{mM}\right.$ [69];
DMFc $D=3.8 \times 10^{-10} \mathrm{~m}^{2} \mathrm{~s}^{-1}, c^{*}=0.5 \mathrm{mM}$, determined by steadystate measurements (results not shown)), the theoretical $i_{\mathrm{T}, \infty}$ can be calculated to be equal to 10.2 and $0.43 \mathrm{nA}$, respectively. The experimental steady-state currents recorded at $10 \mathrm{mV} \mathrm{s}^{-1}$ shown current values that are close to the calculated ones, in accordance to the experimental RG values. Despite of this, the observed current values are in all cases bigger than the calculated ones, most likely due to a slightly protruded active electrode area generated from the blade cutting process as can be seen from Fig. 1. In fact as shown by Zoski and Mirkin this is an important factor, since higher currents are expected for protruded electrodes than for inlaid ones having the same radius [70]. Similar results have been obtained by Burt and Unwin with carbon fiber electrodes coated with a very thin layer of polyoxyphenylene and exposed by blade cutting [53]. To avoid such limitations, the improvement of the active electrode area exposition by blade cutting might be envisaged (e.g. by developing a guillotine system that allows a smoother and more homogeneous cut). Additional factors that influence the response of the probes as described by Cornut et al. are the possible geometrical deviations from ideal disk microelectrodes (e.g. recessed electrodes, off-centered electrodes and elliptic active electrode areas) [44]. Since it is difficult to achieve perfect microdisk probes, independent from the probe fabrication methodology, it is important to establish clearly the effect of such geometrical deviations. For instance, slight deviations from perfect disk probes to elliptic active electrode areas are a common result when preparing microelectrodes. In the case of the probe shown in Fig. 1f, the elliptic deviation corresponds to a value equal to 0.95 (i.e. ratio between the thinnest and widest radius of the active electrode area). According to Cornut et al., this could lead to a difference equal to $4.8 \%$ in the calculated current by using Eq. (1) and the parameters employed for the cyclic voltammetry shown in Fig. 2a [44].

In the case of the experiments performed in DCE, evaporation of the solvent might also contribute to the observed difference. It is important to highlight that Parylene $C$ presents a high chemical stability and is not soluble in organic solvents at room temperature (the solubility increase only after $150^{\circ} \mathrm{C}$ ), for instance during the complete time scale of the different experiments performed in DCE (i.e. cyclic voltammetries, SECM approach curves), there was no change on the recorded current due to coating failure such ill steady state response or drastically increase of the recorded current. Moreover, no planar diffusion behavior was observed for all the tested scan rates (i.e. from $10 \mathrm{mV} \mathrm{s}^{-1}$ to $100 \mathrm{mV} \mathrm{s}^{-1}$ ), corroborating that the electrode is not recessed and it is well sealed by the Parylene C film.

To completely characterize the electrochemical behavior of the Parylene C coated probes, SECM approach curves over insulating (i.e. glass) and conductive (i.e. Pt film) substrates were performed (see Fig. 3a). The current profile observed during the approach curve over the insulating substrate showed a smaller current decrease expected for microelectrodes with small RG values where the blocking of the redox species diffusion toward the electrode surface is counterbalanced by the diffusion of species from back the microelectrode plane.

On the contrary, when approaching over a conductive substrate an enhanced increase on the feedback response is observed due to the fact that the electrode can be brought into a more close position to the sample substrate before touching it. As it can be seen in Fig. 3a, a clear jump on the current is observed at very close normalized working distances $\left(\approx 0.2 \mathrm{~L}=d / r_{\mathrm{T}}\right)$, as the microelectrode surface is physically connected with the Pt substrate with a higher surface area. The latter not only confirms that the microelectrode might be slightly protruded (vide supra), but also the flexibility of the Parylene $C$ coated microelectrodes to bend until the microelectrode is in direct contact with the substrate. Similar results were recently published by Shen et al., where a glass microelectrode was 

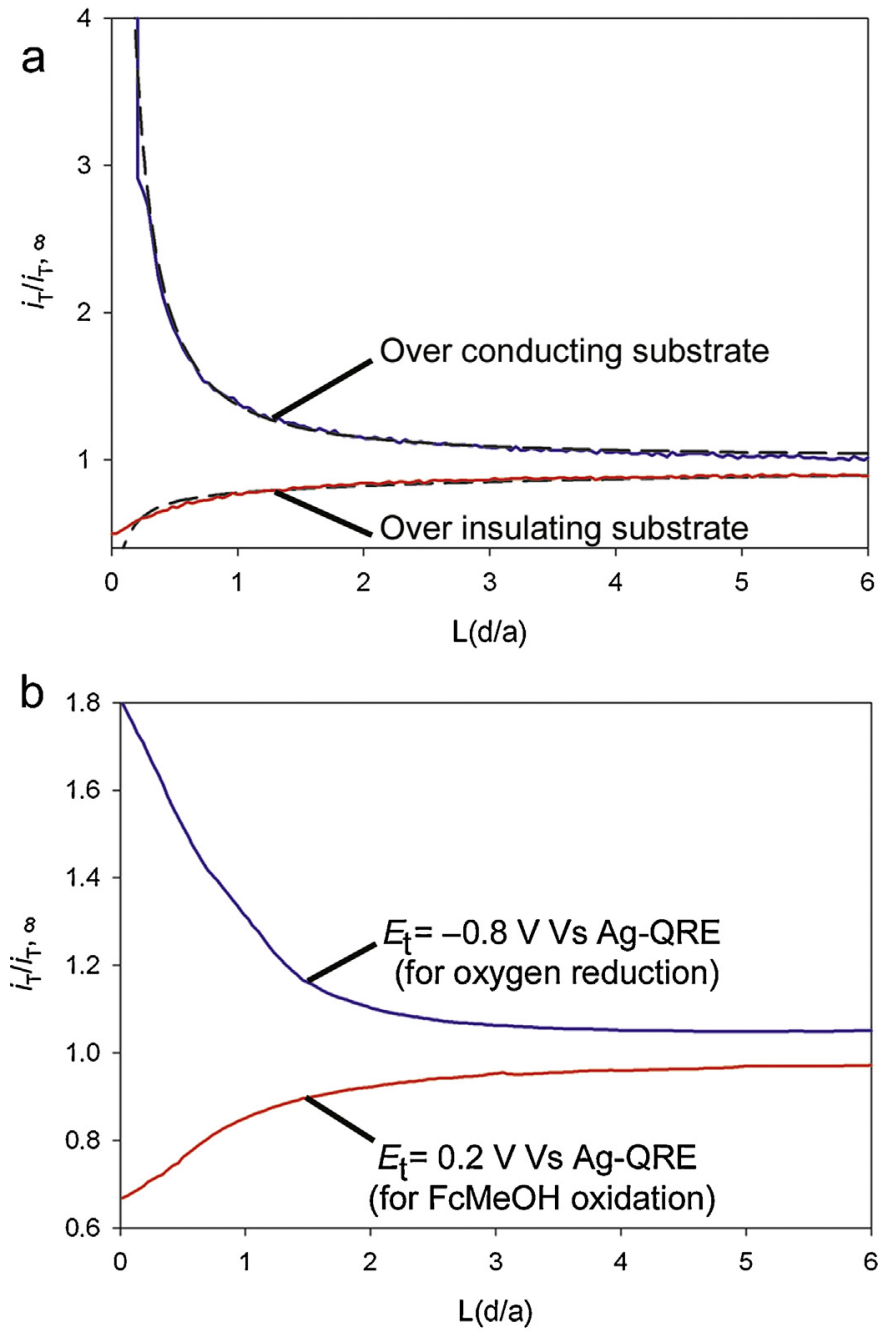

Fig. 3. (a) Approach curve to an insulating (glass, continuous line) and conductive (Pt film, continuous line) substrate with a Parylene $C$ coated Pt microelectrode $\left(r_{\mathrm{T}}=12.5 \mu \mathrm{m}, \mathrm{RG}=1.2\right)$ biased at a tip potential $\left(E_{\mathrm{t}}\right)$ equal to $0.2 \mathrm{~V}$ vs. Ag-ORE. (b) Approach curves to an air/liquid interface by using a submarine Parylene $C$ coated $\mathrm{Pt}$ microelectrode $\left(r_{\mathrm{T}}=12.5 \mu \mathrm{m}, \mathrm{RG}=1.2\right.$, see Fig. $\left.1 \mathrm{a}\right)$ biased at $E_{\mathrm{t}}=0.2 \mathrm{~V}(\mathrm{FcMeOH}$ oxidation) and $E_{\mathrm{t}}=-0.8 \mathrm{~V}$ (oxygen reduction) vs. Ag-QRE, respectively. Other working conditions: $2 \mathrm{mM}$ FcMeOH in $\mathrm{KNO}_{3} 0.1 \mathrm{M}$. Counter electrode $=\mathrm{Pt}$ wire, quasireference electrode $=\mathrm{Ag}$ wire. Theoretical positive and negative feedback approach curves are depicted as black dashed lines [20]. Translation speed $=1 \mu \mathrm{m} / \mathrm{s}$.

mechanical polished until a protruded probe with a small RG value $(<1.1)$ was obtained [46]. In this case, striking enhancement on the positive feedback response (c.a. 15-30 times) was obtained only after judicious alignment between the microelectrode plane and the sample surface. It is important to notice that in the present work, no efforts were made in order to align the sample and probe planes. Despite of that, a very close proximity between the active electrode area of the probe and the sample can be achieved without crashing the microelectrode or destroying the sample. By fitting the experimental approach curve with the theoretical one for the positive feedback, for instance, it can be determined that the Pt disc was about $2.5 \mu \mathrm{m}$ from the sample substrate before touching it. Considering the probe size and the presence of a possible probe-substrate misalignment, this working distance is indeed small and allows for an enhanced positive feedback response. Moreover, it will permit electrochemical studies in physically difficult to reach microenvironments without perturbing their geometry or properties (e.g. droplets or living cells) [53].
To further show the advantages of the present strategy, a probe previously shaped as a hook was employed as a submarine electrode to study mass transport processes at an air/liquid interface (see Fig. 3b). With this aim, the microelectrode was previously submerged totally in a solution of FcMeOH $2 \mathrm{mM}$ in $\mathrm{KNO}_{3} 0.1 \mathrm{M}$. Then the probe was retracted vertically toward the air/liquid interface while biasing the microelectrode to a potential where the mass transport controlled oxidation of $\mathrm{FcMeOH}$ (i.e. $0.2 \mathrm{~V}$ vs. Ag QRE) or reduction of oxygen (i.e. $-0.8 \mathrm{~V}$ vs. Ag QRE) took place. As it can be seen in Fig. 3b, a decrease on the current is observed when the probe is approaching the air/liquid interface due to the blocking of the FcMeOH species diffusion to the surface of the microelectrode. On the other hand, a current increase is recorded when approaching the air/liquid interface with the probe biased to $-0.8 \mathrm{~V} \mathrm{vs.} \mathrm{Ag} \mathrm{QRE.}$ This is due to the fast transfer of oxygen from air into the solution to replace the oxygen depleted at the probe tip [71]. Notice that the air/liquid interface can be deformed during the approach curve due to the presence of the micrometer probe in its vicinity, and even drag a small portion of liquid into the other phase [72]. As a result more complicate determination of the interface position and the current profile requires, for instance, special attention when fitting theoretical and experimental approach curves. Ciani et al. obtained similar results when studying the transfer of oxygen through a lipid membrane supported at an air/liquid interface by using a submarine mercury hemispherical microelectrode [72]. In fact, it was shown both experimentally and theoretically that the mechanical perturbations of the air/liquid interface by the approaching probe is a reproducible process that depends on the counterbalance between surface tension of the water phase and the features of the moving microelectrode [72]. In summary, several charge transfer and mass transport processes taking place at and from almost any interface can be studied by using the Parylene $C$ coated microelectrodes.

Finally, the Parylene $C$ coated microelectrodes were employed for SECM imaging of Pt on glass patterns in contact and contactless mode. Fig. 4a shows several line scans performed over two Pt bands (i.e. $50 \mu \mathrm{m}$ width and $50 \mu \mathrm{m}$ pitch) at different probe-substrate positions. As it is expected, higher current contrast between the conductive and insulating areas are obtained when the probe is closer to the sample substrate. Indeed, when the probe is brought into physical contact with the substrate $\left(h_{\mathrm{P}} \leq 0\right)$, the highest current contrast is achieved. In order to avoid any misunderstanding with common SECM conventions (i.e. sample penetration for $d<0$ ), the probe-substrate distance $(d)$ was redefined in terms of a new parameter $\left(h_{\mathrm{P}}\right)$ as reported previously [21]. Briefly, $d$ can be defined as $d=h_{\mathrm{P}}+t_{\mathrm{L}} \sin (\alpha)$, where $t_{\mathrm{L}}$ is the thickness of the polymeric film that is between the sample surface and the active electrode area (e.g. Pt wire), $h_{\mathrm{P}}$ is the difference between the height of the attachment point of the Parylene $C$ coated microelectrode with respect to the sample surface $\left(h_{\mathrm{A}}\right)$ and the length of the probe in the unbent state $\left(l_{\mathrm{T}}\right)\left(\right.$ i.e. $\left.h_{\mathrm{P}}=h_{\mathrm{A}}-l_{\mathrm{T}}\right)$ and $\alpha$ the tilt angle established between the sample surface and the probe when working in contact mode. Thus, $d$ will always have positive values, while $h_{\mathrm{P}}$ will become negative when physical contact is established. Notice that the electrochemical response of the Parylene $C$ coated microelectrodes is not affected when the probe is bent upon contact and further scanning over the substrate. Indeed, Rui et al. [56]. have shown that Parylene C coated Pt microelectrodes can be bent $90^{\circ} \mathrm{C}$ without a drastically detriment of the electrical properties of the Pt wire or insulating features of the Parylene $C$ coating.

The line scans obtained in contact mode were corrected along their $x$ axis as the bending of the probe introduce a shift on the real position of the probe. The latter does not represent a problem as long as the direction and bending of the probe is maintained constant during the whole SECM imaging process. Although, the 
a
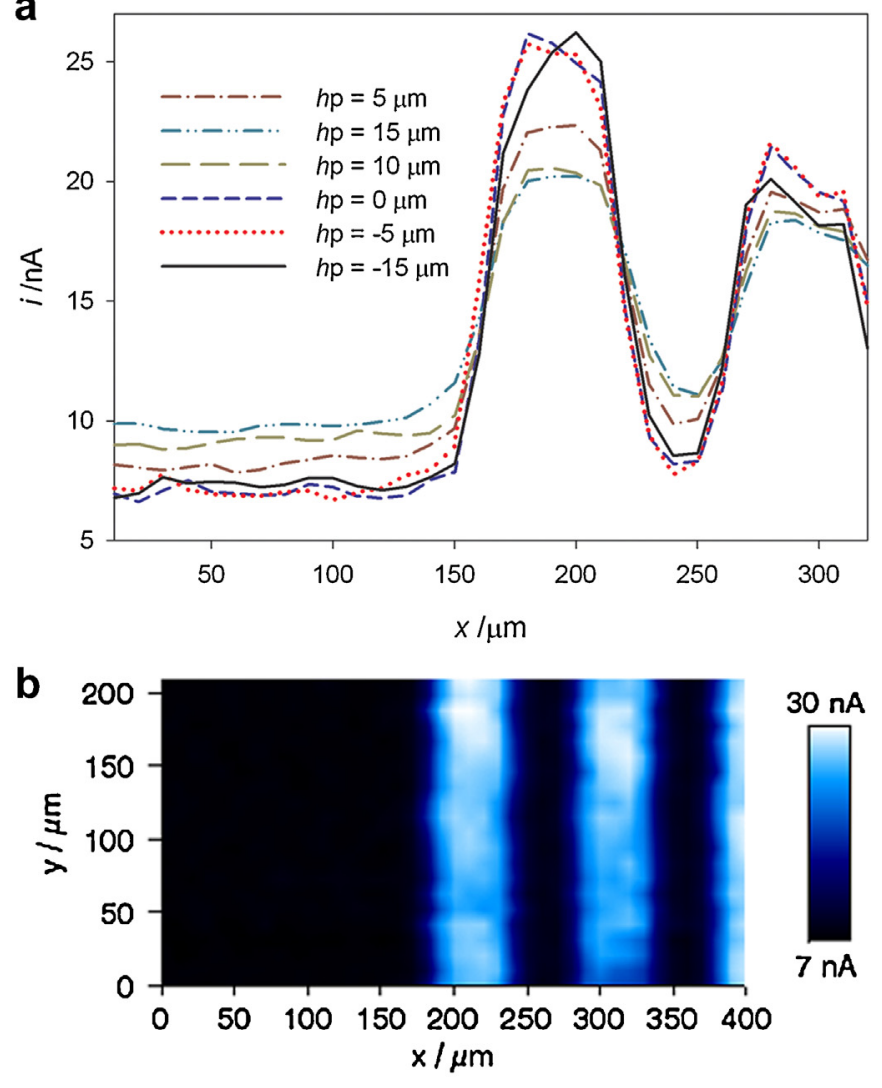

C

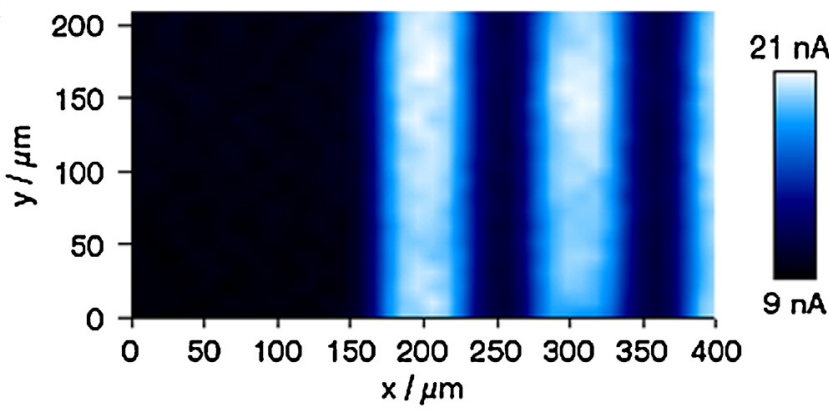

Fig. 4. (a) SECM line scans at different working distances $\left(h_{\mathrm{P}}=15 \mu \mathrm{m}\right.$ dash-dotdotted line; $h_{\mathrm{P}}=10 \mu \mathrm{m}$ dashed line; $h_{\mathrm{P}}=5 \mu \mathrm{m}$ dash-dotted line; $h_{\mathrm{P}}=0 \mu \mathrm{m}$ dashed line; $h_{\mathrm{P}}=-5 \mu \mathrm{m}$ dotted line; $h_{\mathrm{P}}=-15 \mu \mathrm{m}$ continuous line). SECM images of a Pt on glass bands with a Parylene $C$ coated Pt microelectrode $\left(r_{\mathrm{T}}=12.5 \mu \mathrm{m}, \mathrm{RG}=1.5\right)$ in (b) contact and (c) contact-less mode. For (b) $h_{\mathrm{P}}=-10 \mu \mathrm{m}$. A lift-off routine was employed during all the reverse scans. For (c) $h_{\mathrm{P}}=10 \mu \mathrm{m}$. Other working conditions: $2 \mathrm{mM}$ ferrocene methanol in $\mathrm{KNO}_{3} 0.1 \mathrm{M}$. Step size $=25 \mu \mathrm{m}$, translation speed $=25 \mu \mathrm{m} / \mathrm{s}$. The Pt bands width and pitch were equal to $50 \mu \mathrm{m}$.

SECM image obtained in contact mode $\left(h_{\mathrm{P}}=-10 \mu \mathrm{m}\right)$ shows a high current contrast and a Pt band width close to the real one, local reactivity information is missed during the scanning process (i.e. dark zones on the Pt band regions) due to the perturbation of the degree and direction of the probe bending (see Fig. 4b). For this reason, in order to avoid such situation, it is advisable to employ the Parylene $C$ coated microelectrodes in a contact-less mode for imaging purposes, even though the current contrast is not as high as the one obtained in contact mode (see Fig. 4c). For local amperometric monitoring of redox species being generated or consumed at the specimen surface, it might be beneficial to work with the Parylene $C$ coated probes in a contact mode in order to reach a higher sensitivity, while avoiding any sample or probes destruction.

\section{Conclusions}

This technical report presents a soft SECM microelectrode prepared by following a simple and fast protocol based on the Parylene $C$ coating of a Pt wire or a carbon fiber. The success on probe preparation was equal to $90 \%$, while low expertise and preparation time was required. The obtained microelectrodes present a small and constant RG value, which can be obtained reproducibly after blade cutting a cross-section of the Parylene $C$ coated wire previously protected with a low melting point wax. After complete wax dissolution in acetone, a round and well-centered active electrode area is achieved. Despite the fact that the active electrode area is slightly protruded and does not present a highly smooth surface, the high quality electrochemical response recorded in cyclic voltammetries, SECM approach curves and SECM imaging over heterogeneous interfaces confirmed the applicability of these flexible probes for SECM studies in aqueous and organic media, as well as, in microenvironments where small working distances are required.

\section{Acknowledgements}

The Fonds National Suisse pour la Recherche Scientifique (Grant no. 20PA21_121570/1) and Deutsche Forschungsgemeinschaft (Wi $1617 / 10$ ) are thanked for financial support of the binational project "High throughput SECM imaging". H. Deng thanks the China Scholarship Council (CSC) for financial support and P. Peljo is grateful for the financial support by Academy of Finland (Grant no. 133261) and Magnus Ehrnrooth foundation. The authors thank Professor Gunther Wittstock and Dr. Andreas Lesch from the University of Oldenburg for the development of the lift-off routine integrated into the SECMx software and employed here for the SECM imaging experiments in contact mode. The technical assistance by Cyrille Hibert, Yvan Deillon and Danièle Laub is also acknowledged.

\section{References}

[1] G. Wittstock, R. Hesse, W. Schuhmann, Patterned self-assembled alkanethiolate monolayers on gold. Patterning and imaging by means of scanning electrochemical microscopy, Electroanalysis 9 (1997) 746-756.

[2] T. Yasukawa, T. Kaya, T. Matsue, Characterization and imaging of single cells with scanning electrochemical microscopy, Electroanalysis 12 (2000) 653-659.

[3] I. Beaulieu, S. Kuss, J. Mauzeroll, M. Geissler, Biological scanning electrochemical microscopy and its application to live cell studies, Analytical Chemistry 83 (2011) 1485-1492.

[4] M. Zhang, H.H. Girault, SECM for imaging and detection of latent fingerprints, Analyst 134 (2009) 25-30.

[5] F. Cortés-Salazar, M. Zhang, A. Becue, J.-M. Busnel, M. Prudent, C. Champood, H.H. Girault, Human fingerprint imaging by scanning electro-chemical microscopy (SECM), Chimia 63 (2009) 580.

[6] K. Fushimi, M. Seo, Trial for evaluation of heterogeneity of passive film on iron by a scanning electrochemical microscope, Zairyo to Kankyo 46 (1997) 797-803

[7] A.M. Simões, A.C. Bastos, M.G. Ferreira, Y. González-García, S. González, R.M. Souto, Use of SVET and SECM to study the galvanic corrosion of an iron-zinc cell, Corrosion Science 49 (2007) 726-739.

[8] D. Mandler, Micro- and nanopatterning using the scanning electrochemical microscope, in: A.J. Bard, M.V. Mirkin (Eds.), Scanning Electrochemical Microscopy, Marcel Dekker, New York, Basel, 2001, pp. 593-627.

[9] C. Slim, Y. Tran, M.M. Chehimi, N. Garraud, J.P. Roger, C. Combellas, F. Kanoufi, Microelectrochemical patterning of surfaces with polymer brushes, Chemistry of Materials 20 (2008) 6677-6685.

[10] D.A. Walsh, J.L. Fernandez, A.J. Bard, Rapid screening of bimetallic electrocatalysts for oxygen reduction in acidic media by scanning electrochemical microscopy, Journal of the Electrochemical Society 153 (2006) E99-E103.

[11] A.J. Bard, Inner-sphere heterogeneous electrode reactions. Electrocatalysis and photocatalysis: the challenge, Journal of the American Chemical Society 132 (2010) 7559-7567.

[12] R.C. Engstrom, M. Weber, D.J. Wunder, R. Burgess, S. Winquist, Measurements within the diffusion layer using a microelectrode probe, Analytical Chemistry 58 (1986) 844-848

[13] H.Y. Liu, F.R.F. Fan, C.W. Lin, A.J. Bard, Scanning electrochemical and tunneling ultramicroelectrode microscope for high-resolution examination of electrode 
surfaces in solution, Journal of the American Chemical Society 108 (1986) 3838-3839.

[14] A.L. Barker, M. Gonsalves, J.V. MacPherson, C.J. Slevin, P.R. Unwin, Scanning electrochemical microscopy: beyond the solid/liquid interface, Analytica Chimica Acta 385 (1999) 223-240.

[15] J. Kwak, A.J. Bard, Scanning electrochemical microscopy. Theory of the feedback mode, Analytical Chemistry 61 (1989) 1221-1227.

[16] K. Eckhard, X. Chen, F. Turcu, W. Schuhmann, Redox competition mode of scanning electrochemical microscopy (RC-SECM) for visualisation of local catalytic activity, Physical Chemistry Chemical Physics 8 (2006) 5359-5365.

[17] J. Rodríguez-López, M.A. Alpuche-Avilés, A.J. Bard, Interrogation of surfaces for the quantification of adsorbed species on electrodes: oxygen on gold and platinum in neutral media, Journal of the American Chemical Society 130 (2008) $16985-16995$.

[18] C. Lee, J. Kwak, F.C. Anson, Application of scanning electrochemical microscopy to generation/collection experiments with high collection efficiency, Analytical Chemistry 63 (1991) 1501-1504.

[19] C.J. Slevin, J.V. Macpherson, P.R. Unwin, Measurement of local reactivity at liquid/solid, liquid/liquid, and liquid/gas interfaces with the scanning electrochemical microscope: principles, theory, and applications of the double potential step chronoamperometric mode, Journal of Physical Chemistry B 101 (1997) 10851-10859.

[20] R. Cornut, C. Lefrou, New analytical approximation of feedback approach curves with a microdisk SECM tip and irreversible kinetic reaction at the substrate, Journal of Electroanalytical Chemistry 621 (2008) 178-184.

[21] F. Cortés-Salazar, M. Traeuble, F. Li, J.-M. Busnel, A. Gassner, M. Hojeij, G. Wittstock, H.H. Girault, Soft stylus probes for scanning electrochemical microscopy, Analytical Chemistry 81 (2009) 6889-6896.

[22] C. Wei, A.J. Bard, G. Nagy, K. Toth, Scanning electrochemical microscopy. 28: ion-selective neutral carrier-based microelectrode potentiometry, Analytical Chemistry 67 (1995) 1346-1356.

[23] D.O. Wipf, A.J. Bard, Scanning electrochemical microscopy. 15: improvements in imaging via tip-position modulation and lock-in detection, Analytical Chemistry 64 (1992) 1362-1367.

[24] D.O. Wipf, A.J. Bard, D.E. Tallman, Scanning electrochemical microscopy. 21: constant-current imaging with an autoswitching controller, Analytical Chemistry 65 (1993) 1373-1377.

[25] J.V. Macpherson, P.R. Unwin, Combined scanning electrochemical-atomic force microscopy, Analytical Chemistry 72 (2000) 276-285.

[26] C. Kranz, G. Friedbacher, B. Mizaikofft, A. Lugstein, J. Smoliner, E. Bertagnolli, Integrating an ultramicroelectrode in an AFM cantilever: combined technology for enhanced information, Analytical Chemistry 73 (2001) 2491-2500.

[27] O. Sklyar, A. Kueng, C. Kranz, B. Mizaikoff, A. Lugstein, E. Bertagnolli, G. Wittstock, Numerical simulation of scanning electrochemical microscopy experiments with frame-shaped integrated atomic force microscopy-SECM probes using the boundary element method, Analytical Chemistry 77 (2005) $764-771$.

[28] T.H. Treutler, G. Wittstock, Combination of an electrochemical tunneling microscope (ECSTM) and a scanning electrochemical microscope (SECM): application for tip-induced modification of self-assembled monolayers, Electrochimica Acta 48 (2003) 2923-2932.

[29] A. Kueng, C. Kranz, A. Lugstein, E. Bertagnolli, B. Mizaikoff, Integrated AFMSECM in tapping mode: simultaneous topographical and electrochemical imaging of enzyme activity, Angewandte Chemie International Edition 42 (2003) 3238-3240.

[30] D.P. Burt, N.R. Wilson, J.M.R. Weaver, P.S. Dobson, J.V. Macpherson, Nanowire probes for high resolution combined scanning electrochemical microscopy atomic force microscopy, Nano Letters 5 (2005) 639-643.

[31] M.M. Lohrengel, A. Moehring, M. Pilaski, Electrochemical surface analysis with the scanning droplet cell, Fresenius Journal of Analytical Chemistry 367 (2000) 334-339.

[32] T.W. Spaine, J.E. Baur, A positionable microcell for electrochemistry and scanning electrochemical microscopy in subnanoliter volumes, Analytical Chemistry 73 (2001) 930-938.

[33] F. Turcu, A. Schulte, W. Schuhmann, Scanning electrochemical microscopy (SECM) of nanolitre droplets using an integrated working/reference electrode assembly, Analytical and Bioanalytical Chemistry 380 (2004) $736-741$.

[34] F. Cortés-Salazar, A. Lesch, D. Momotenko, J.-M. Busnel, G. Wittstock, H.H. Girault, Fountain pen for SECM, Analytical Methods 2 (2010) 817-823.

[35] F. Cortés-Salazar, D. Momotenko, A. Lesch, G. Wittstock, H.H. Girault, Soft microelectrode linear array for SECM, Analytical Chemistry 82 (2010) 10037-10044.

[36] A.L. Barker, P.R. Unwin, J.W. Gardner, H. Rieley, A multi-electrode probe for parallel imaging in scanning electrochemical microscopy, Electrochemistry Communications 6 (2004) 91-97.

[37] D. Momotenko, F. Cortes-Salazar, A. Lesch, G. Wittstock, H.H. Girault, Microfluidic push-pull probe for scanning electrochemical microscopy, Analytical Chemistry $83 \quad$ (2011) 5275-5282.

[38] D. Momotenko, L. Qiao, F. Cortes-Salazar, A. Lesch, G. Wittstock, H.H. Girault, Electrochemical push-pull scanner with mass spectrometry detection, Analytical Chemistry 84 (2012) 6630-6637.
[39] C.G. Williams, M.A. Edwards, A.L. Colley, J.V. Macpherson, P.R. Unwin Scanning micropipet contact method for high-resolution imaging of electrode surface redox activity, Analytical Chemistry 81 (2009) $2486-2495$.

[40] N. Ebejer, M. Schnippering, A.W. Colburn, M.A. Edwards, P.R. Unwin, Localized high resolution electrochemistry and multifunctional imaging: scanning electrochemical cell microscopy, Analytical Chemistry 82 (2010) 9141-9145.

[41] P. Sun, M.V. Mirkin, Kinetics of electron-transfer reactions at nanoelectrodes, Analytical Chemistry 78 (2006) 6526-6534.

[42] A. Lesch, D. Momotenko, F. Cortes-Salazar, I. Wirth, U.M. Tefashe, F. Meiners, B. Vaske, H.H. Girault, G. Wittstock, Fabrication of soft gold microelectrode arrays as probes for scanning electrochemical microscopy, Journal of Electroanalytical Chemistry 666 (2012) 52-61.

[43] B.B. Katemann, W. Schuhmann, Fabrication and characterization of needle-type Pt-disk nanoelectrodes, Electroanalysis 14 (2002) 22-28.

[44] R. Cornut, A. Bhasin, S. Lhenry, M. Etienne, C. Lefrou, Accurate and simplified consideration of the probe geometrical defaults in scanning electrochemical microscopy: theoretical and experimental investigations, Analytical Chemistry 83 (2011) 9669-9675.

[45] K. Aoki, C. Zhang, J. Chen, T. Nishiumi, Fabrication of glass-coated electrodes with nano- and micrometer size by means of dissolution with HF, Electrochimica Acta 55 (2010) 7328-7333.

[46] M. Shen, N. Arroyo-Curras, A.J. Bard, Achieving nanometer scale tipto-substrate gaps with micrometer-size ultramicroelectrodes in scanning electrochemical microscopy, Analytical Chemistry 83 (2011) 9082-9085.

[47] K. Potje-Kamloth, J. Janata, M. Josowicz, Electrochemically prepared insulation for carbon fiber microelectrodes, Physical Chemistry Chemical Physics 93 (1989) 1480-1485.

[48] A. Schulte, R.H. Chow, A simple for insulating microelectrodes using anodic electrophoretic deposition of paint, Analytical Chemistry 68 (1996) 3054-3058.

[49] C.J. Slevin, N.J. Gray, J.V. MacPherson, M.A. Webb, P.R. Unwin, Fabrication and characterisation of nanometre-sized platinum electrodes for voltammetric analysis and imaging, Electrochemistry Communications 1 (1999) $282-288$.

[50] R.M. Penner, M.J. Heben, N.S. Lewis, Preparation and electrochemical characterization of conical and hemispherical ultramicroelectrodes, Analytical Chemistry 61 (1989) 1630-1636.

[51] P. Sun, Z. Zhang, J. Guo, Y. Shao, Fabrication of nanometer-sized electrodes and tips for scanning electrochemical microscopy, Analytical Chemistry 73 (2001) 5346-5351.

[52] B. Liu, J.P. Rolland, J.M. DeSimone, A.J. Bard, Fabrication of ultramicroelectrodes using A "Teflon-like" coating material, Analytical Chemistry 77 (2005) 3013-3017.

[53] D.P. Burt, P.R. Unwin, Minimally invasive spatial and temporal concentration profiling within working glucose biosensors, Electrochemistry Communications 10 (2008) 934-937.

[54] G.E. Loeb, M.J. Bak, M. Salcman, E.M. Schmidt, Parylene as a chronically stable, reproducible microelectrode insulator, IEEE Transactions on Biomedical Engineering 24 (1977) 121-128.

[55] G.E. Loeb, R.A. Peck, J. Martyniuk, Toward the ultimate metal microelectrode, Journal of Neuroscience Methods 63 (1995) 175-183.

[56] Y.F. Rui, J.Q. Liu, B. Yang, C.S. Yang, D.X. Wei, Mechanical and electrical stability of parylene-based platinum-black coated wire microelectrode for implantable applications, Journal of Applied Electrochemistry, in press

[57] C. Metallo, R.D. White, B.A. Trimmer, Flexible parylene-based microelectrode arrays for high resolution EMG recordings in freely moving small animals, Journal of Neuroscience Methods 195 (2011) 176-184.

[58] D.C. Rodger, W. Li, H. Ameri, A. Ray, J.D. Weiland, M.S. Humayun, Y.C Tai, Flexible parylene-based microelectrode technology for intraocular retinal prostheses, in: Proc. 1st IEEE International Conference on Nano Micro Engineered and Molecular Systems, Zhuhai, 18-21 January, 2006, pp. $743-746$.

[59] E.M. Schmidt, M.J. Bak, P. Christensen, Laser exposure of Parylene-C insulated microelectrodes, Journal of Neuroscience Methods 62 (1995) 89-92.

[60] C.A. Morris, C.C. Chen, L.A. Baker, Transport of redox probes through single pores measured by scanning electrochemical-scanning ion conductance microscopy (SECM-SICM), Analyst 137 (2012) 2933-2938.

[61] B. Su, R.P. Nia, F. Li, M. Hojeij, M. Prudent, C. Corminboeuf, Z. Samec, H.H. Girault, $\mathrm{H}_{2} \mathrm{O}_{2}$ generation by decamethylferrocene at a liquid|liquid interface, Angewandte Chemie International Edition 47 (2008) 4675-4678.

[62] K. Nunes, K. Hallmeier, R. Szargan, T. Raschke, C. Radehaus, G. Wittstock, Evaluation of thin film titanium nitride electrodes for electroanalytical applications, Electroanalysis 19 (2007) 1023-1031.

[63] G. Wittstock, T. Asmus, T. Wilhelm, Investigation of ion-bombarded conducting polymer films by scanning electrochemical microscopy (SECM), Fresenius Journal of Analytical Chemistry 367 (2000) 346-351.

[64] F. Cortes-Salazar, A. Lesch, D. Momotenko, J.-M. Busnel, G. Wittstock, H.H. Girault, Fountain pen for scanning electrochemical microscopy, Analytical Methods 2 (2010) 817-823.

[65] F. Cortes-Salazar, D. Momotenko, H.H. Girault, A. Lesch, G. Wittstock, Seeing big with scanning electrochemical microscopy, Analytical Chemistry 83 (2011) 1493-1499.

[66] F. Cortes-Salazar, D. Momotenko, A. Lesch, G. Wittstock, H.H. Girault, Soft microelectrode linear array for scanning electrochemical microscopy, Analytical Chemistry 82 (2010) 10037-10044. 
[67] A. Lesch, B. Vaske, F. Meiners, D. Momotenko, F. Cortes-Salazar, H.H. Girault, G. Wittstock, Parallel imaging and template-free patterning of self-assembled monolayers with soft linear microelectrode arrays, Angewandte Chemie International Edition 51 (2012) 10413-10416.

[68] C. Lefrou, R. Cornut, Analytical expressions for quantitative scanning electrochemical microscopy (SECM), ChemPhysChem 11 (2010) 547-556.

[69] C.G. Zoski, Ultramicroelectrodes: design, fabrication, and characterization, Electroanalysis 14 (2002) 1041-1051.

[70] C.G. Zoski, M.V. Mirkin, Steady-state limiting currents at finite conical microelectrodes, Analytical Chemistry 74 (2002) 1986-1992
[71] C.J. Slevin, S. Ryley, D.J. Walton, P.R. Unwin, A new approach for measuring the effect of a monolayer on molecular transfer across an air/water interface using scanning electrochemical microscopy, Langmuir 14 (1998) 5331-5334.

[72] I. Ciani, D.P. Burt, S. Daniele, P.R. Unwin, Effect of surface pressure on oxygen transfer across molecular monolayers at the air/water interface: scanning electrochemical microscopy investigations using a mercury hemispherical microelectrode probe, Journal of Physical Chemistry B 108 (2004) 3801-3809. 